\title{
Archéopages
}

Archéopages Archéologie et société

$44 \mid 2017$

Terrains vagues

\section{Les usages du vide. Débat}

Propos recueillis par Catherine Chauveau

Laurence Tranoy, Stéphane Tonnelat et Catherine Chauveau

\section{CpenEdition}

Journals

Édition électronique

URL : https://journals.openedition.org/archeopages/1563

DOI : 10.4000/archeopages. 1563

ISSN : 2269-9872

Éditeur

INRAP - Institut national de recherches archéologiques préventives

Édition imprimée

Date de publication : 1 juin 2017

Pagination : 66-73

ISSN : 1622-8545

Référence électronique

Laurence Tranoy, Stéphane Tonnelat et Catherine Chauveau, « Les usages du vide. Débat », Archéopages [En ligne], 44 | 2017, mis en ligne le 01 juillet 2019, consulté le 04 juin 2021. URL : http:// journals.openedition.org/archeopages/1563; DOI : https://doi.org/10.4000/archeopages.1563 


\section{Les usages du vide}

En se développant, la ville intègre des terrains qui ont eu une fonction définie et contrôlée à un moment, sur lesquels les constructions sont disparues ou abandonnées, qui deviennent ensuite des terrains délaissés, des terrains vides, des terrains vagues. Mais parfois aussi l'urbanisme crée, volontairement ou involontairement, des espaces vides. Ces terrains ont des usages divers, parfois réglementés, parfois marginaux, dont les traces ne se repèrent pas facilement. Mais s'interroger sur ces usages, c'est avoir conscience que le fait urbain ne se réduit pas au fait bâti.

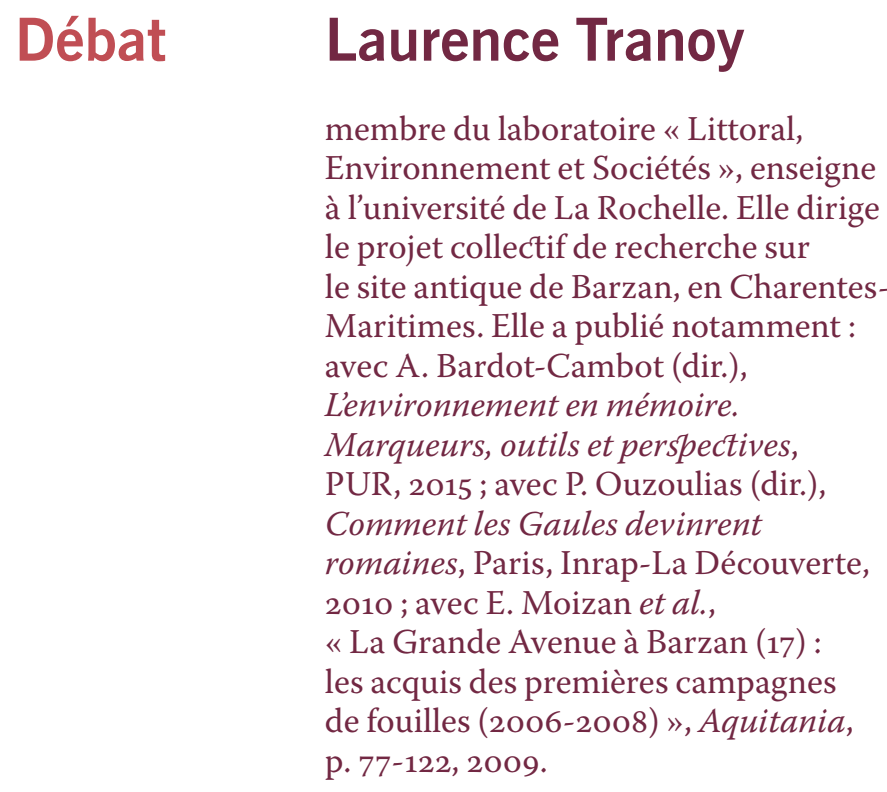

\section{Stéphane Tonnelat}

est chargé de recherche CNRS au laboratoire UMR LAVUE « Laboratoire Architecture, Ville, Urbanisme,

Environnement ». Il mène, dans une approche ethnographique, des études sur certains types de terrains urbains, dont les lieux délaissés. Sur le thème du vide en ville, il a publié notamment : « La carrière d'un interstice urbain à New York. Deux rôles distincts de l'espace dans les mobilisations autour d'un projet d'aménagement », in H. Combes, D. Garibay et

C. Goirand (dir.), Les lieux de la colère. Occuper l'espace pour contester, de Madrid à Sanaa, Karthala, 2016 ; Interstices urbains. Paris-New York. Entre contrôles et mobilités, quatre espaces résiduels de l'aménagement, thèse consultable en ligne, 2003 ; «Interstices urbains, les mobilités des terrains délaissés de l'aménagement ", Chimères, ${ }^{\circ}$ 52, 2003, p. 134-151. 


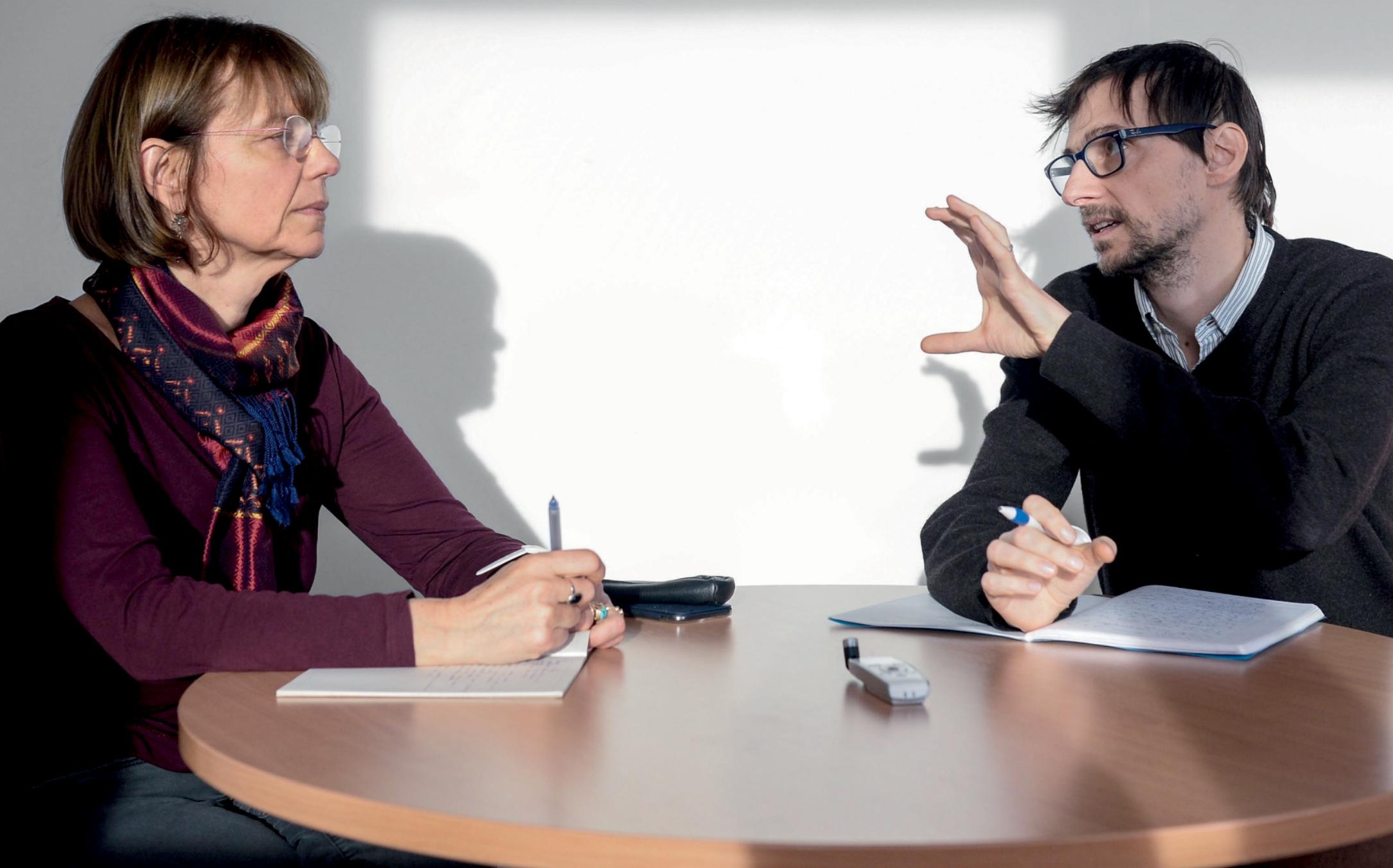


Laurence Tranoy En archéologie, nous sommes souvent confrontés à des espaces que nous n'arrivons pas à identifier parce que nous ne trouvons pas de marqueurs immédiats. Ce qui peut les caractériser, c'est d'être entourés par des éléments que nous reconnaissons, en revanche, parfaitement; et c'est cela qui nous les fait qualifier d'espaces vides. Je me suis interrogée sur ce fait notamment sur deux lieux, où se sont révélés des espaces de statut différent. Le premier est Barzan, petite ville antique en bordure de l'estuaire de la Gironde, au rôle économique plutôt que politique. C'était un port qui devait être bien visible de loin, doté d'une architecture publique ostentatoire : un grand temple et une longue colonnade qui devait servir de fond de ciel pour les bateaux. En avant de ce dispositif se trouve un espace trapézoïdal d'un peu plus de deux hectares et demi. Les prospections aériennes anciennes laissant penser qu'il y avait des vestiges, nous avons fait des diagnostics, qui se sont révélés négatifs. Je doutais qu'il y ait eu, en cour de ville, un lieu sans usage et, partant de l'hypothèse que cet endroit avait pu être stabilisé, j'ai fait appel à des chercheurs ${ }^{\mathbf{1}}$ qui ont une autre perception des sédiments. Il est apparu qu'il y avait eu un aménagement végétal, des allées et des surfaces piétinées. L'espace vide est devenu une aire de rassemblement, de procession : un espace occupé, organisé. Le second est un ensemble funéraire antique à Lyon. On a constaté qu'il existait une zone "sans rien ", alors que le nombre des tombes et leurs recoupements étaient importants. Là, c'est la corrélation entre la présence d'un aqueduc et une inscription interdisant l'occupation de l'espace destiné à protéger le monument qui explique ce constat ; la nécessité de pouvoir accéder à un ouvrage public, surveillé et entretenu, crée une zone non aedificandi. On se dit que ce genre de lieux finit par devenir des terrains vagues, une place à prendre pour des personnes qui n'en ont pas.

Stéphane Tonnelat Nous nous posons bien la même question : qu'est-ce que cela veut dire un terrain vide et comment le reconnaître? Mes premières études m'ont fait constater qu'au sein de la planification urbaine la plus pensée, il peut y avoir des zones impensées. J'ai commencé mes recherches à Évry, l'une des cinq villes nouvelles construites autour de Paris pour la désenclaver, selon les principes hérités de l'urbanisme moderne ${ }^{2}$, chacune avec un schéma différent. Évry est une ville posée comme un ovni : elle ne respecte pas la trame viaire, ni la trame agricole ni celle des propriétés et elle n'englobe nullement le vieux village. C'est une rencontre radicale entre un nouveau type d'urbanisme très planifié et un existant rural complètement nié. Le fait que la Nationale coupe la ville sans se raccorder à sa trame urbaine a créé notamment toute une série d'espaces résiduels. Je les ai appelés les interstices urbains et définis comme des productions de jonction entre des formes d'aménagement d'époques et de natures archéologues des jardins.

2. Dans la lignée des

idées de Le Corbusier

et de la charte

d'Athènes (1933) différentes. J'ai distingué deux types : les lieux résiduels dus à des restes d'aménagements contemporains, les « interstices résiduels synchrones »; les terrains qui ont eu un usage et qui l'ont perdu, les « interstices résiduels diachroniques ». Je me suis ensuite intéressé à Charenton-le-Pont, à l'est de Paris, car c'est une ville traversée par divers réseaux de l'urbanisme des $\mathrm{XIX}^{\mathrm{e}}$ et $\mathrm{XX}^{\mathrm{e}}$ siècles : comme frontière nord, le bois de Vincennes ; en plein milieu, les voies ferrées de la gare de Lyon; le long de la Seine, la frontière sud, l'autoroute $\mathrm{A}_{4}$ et des pipelines ; vers l'ouest, le périphérique, construit dans la zone non aedificandi des dernières murailles de Paris. Cette ville est ponctuée de petits restes, qui n'ont pas vraiment d'existence sur la carte ; par exemple, la voie aménagée sur la bande de terrain entre l'autoroute et la Seine est la seule rue de la ville qui n'a pas de nom. Cette rue est cependant d'importance puisqu'elle est née de contraintes techniques liées d'une part à la Seine (l'ancienne servitude de halage, qui nécessite un accès libre pour le passage sur la berge), d'autre part à l'autoroute, qui doit être bordée par une distance de non aedificandi, variable dans sa largeur (autour de quelques dizaines de mètres) où sont enterrés les pipelines. On voit que la relation est très forte entre les grands aménagements urbains, essentiellement les infrastructures de transport, et la création de restes urbains, qui ont bien une fonction, sauf qu'elle ne relève pas de l'usage de l'espace par les habitants ; c'est une fonction qu'on pourrait appeler tectonique, liée au terrain même.

Il y a beaucoup de restes urbains dans les grandes villes, notamment des friches industrielles, même si celles-ci tendent à disparaître puisqu'on les transforme peu à peu. Ces friches sont généralement liées aux infrastructures de transport, l'industrie s'étant, à partir du XIX ${ }^{\mathrm{e}}$ siècle, développée le long des voies ferrées, des routes et des fleuves, en y aménageant notamment des aires de stockage construites ou de plein air. L'abandon progressif de ces terrains industriels a donné lieu à ce qu'on a nommé des terrains vagues, une notion, en urbanisme, qui date du $\mathrm{Xx}^{\mathrm{e}}$ siècle. Les autres espaces urbains vides sont des espaces publics. Ils ont des fonctions réglementées de circulation, de marché, de promenade, de jeu, de rassemblement... Ils ont des noms et des signalements sur les cartes et sont facilement accessibles. Mais pendant très longtemps en urbanisme, ces espaces ont été considérés comme des restes urbains, car ils proviennent souvent de zones qui subsistent entre les constructions et auxquelles on attribue des usages a posteriori. Chacun peut le constater dans sa ville : de nombreuses voies et places anciennes ne sont pas vraiment dessinées ; les squares urbains sont souvent des restes de la rencontre de la trame avec des accidents géographiques ou topologiques, ce qui crée des endroits plus difficiles à construire, 


\section{La qualification de « vide» est un} jugement péjoratif et c'est cela qui vide le terrain de sens.

Stéphane Tonnelat
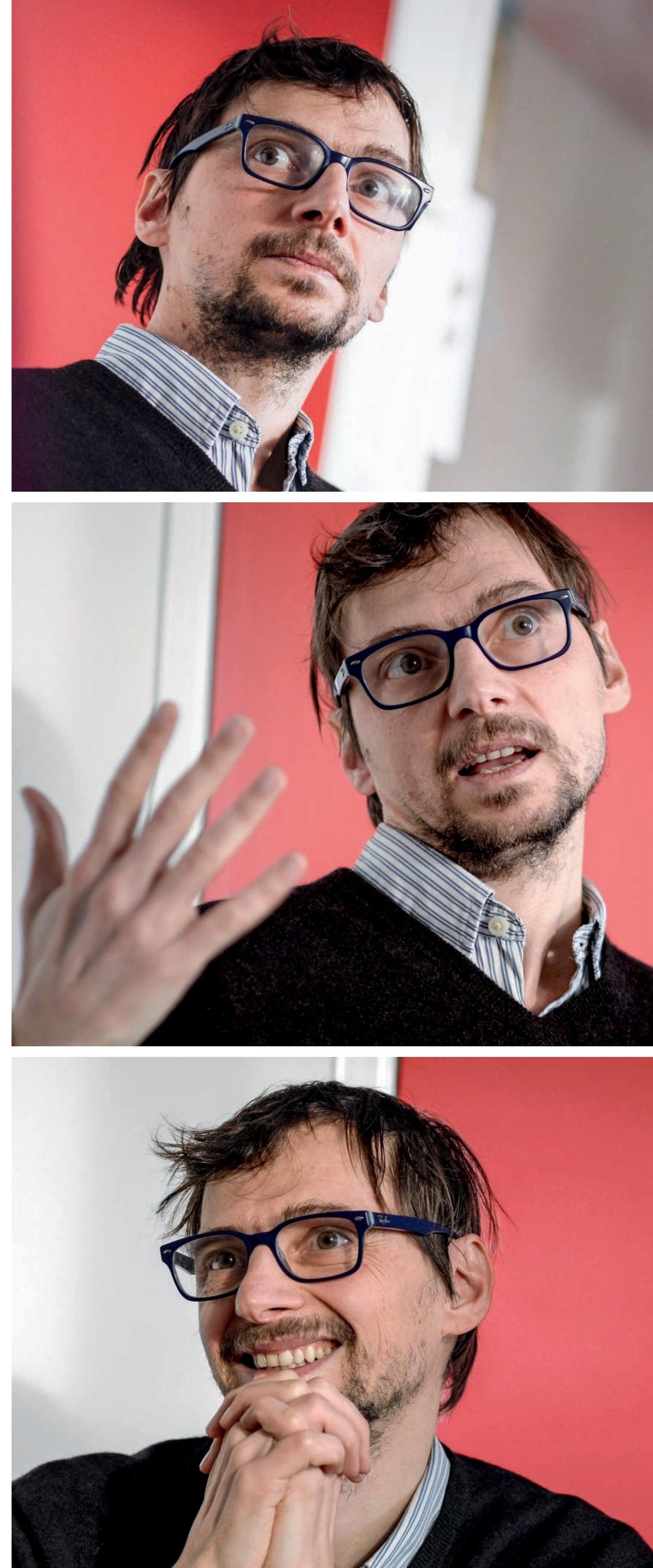
à cause de contraintes ou à cause de formes un peu bizarres. Et puis, il y a les dents creuses, des parcelles sur lesquelles le bâtiment a disparu, ce qui crée un « trou » dans le parcellaire et dans l'alignement sur rue. L'autre créateur de vide est le modernisme architectural. Construire des tours ou des barres pour augmenter la densité résidentielle sur une faible portion des parcelles, dans une volonté de laisser une part aux jardins et de donner la sensation d'avoir de la place, fait disparaître la rue et les alignements de bâtis. La voie est prévue mais elle n'a plus de profil, et cela nous fait bien comprendre à quel point la rue n'était pas pensée comme l'aspect important du quartier. C'est le cas à Paris, dans des quartiers des XII ${ }^{\mathrm{e}}$ et XIII ${ }^{\mathrm{e}}$ arrondissements rénovés dans les années 1970, et surtout en banlieue où il n'y avait pas encore une assez forte densité de bâti pour préserver la rue. Après les années 1980, on a réalisé que la rue était en fait dessinée par les pleins et que, quand on enlevait ces pleins, on la perdait. On a alors voulu revaloriser la rue comme un lieu d'usage, comme une forme construite ${ }^{3}$. Car dans nos professions, finalement, on s'intéresse en priorité au bâti avant de regarder ce qui est entre les bâtis. Ce sont pourtant des espaces essentiels mais on n'en a pris conscience que très récemment, depuis que les études de morphologie urbaine des années 1970 et 1980 ont montré que les trames viaires urbaines sont ce qu'il y a de plus durable ; le moins durable, ce sont les bâtiments ${ }^{4}$, n'en déplaisent aux architectes, et puis les lots, les parcelles. Mais j'imagine que les archéologues portent une attention toute particulière à ces espaces qui se créent en négatif.

LT Oui, mais on ne prend pas assez en compte ces espaces vides et ce qu'ils nous apprendraient $\mathrm{du}$ fonctionnement social urbain, pour diverses raisons. D'abord, nous avons, en quelque sorte, un biais d'étude : les espaces vides donnent des diagnostics négatifs, sur lesquels, donc, l'État ne prescrit pas. Ensuite, ce champ de la recherche est moins nourri car la dynamique de recherche en archéologie urbaine a été ralentie par l'État. Enfin, même si on raisonne beaucoup sur les structures en négatif, on peut aussi facilement se focaliser sur le bâti, qu'il soit présent ou absent. Votre notion d'interstices résiduels synchrones et diachroniques me semble très intéressante pour nos réflexions. « Interstices synchrones » pourrait s'appliquer aux espaces qui bordent les voies antiques, dont on ne distingue pas tous les usages, entre circulation, étals, services ${ }^{\mathbf{5}}$... Encore une fois parce que les prescriptions portant sur les voies se concentrent souvent sur la bande roulante en oubliant que la voie est tout un ensemble. En revanche, pour revenir à l'urbain, l'importance de la rue et sa persistance dans la morphologie urbaine sont des évidences en archéologie.

Les axes antiques perdurent souvent dans le tissu urbain, voire rural. Et la ville antique nous paraît fondée sur la rue. Et puis il existe un mot antique, l'urbanitas, qui se réfère à la fois à la ville et à la manière d'y vivre.

ST C'est cela, regarder la manière d'y vivre, c'est la seule façon, selon moi, de regarder les interstices et les espaces publics. On s'aperçoit alors qu'ils ne sont pas vides mais très utilisés. Les espaces vides le sont seulement au regard de la production urbaine, de la planification d'une ville, mais, en fait, ils ont des usages et des fonctions bien réels. Mais il est vrai que cela ne se voit pas sur un plan. Parmi les espaces vides, il faut compter aussi les espaces en attente de construction. Dans un projet d'urbanisme actuel, environ dix ans se passent du moment où l'on fait le plan à celui où l'on livre les bâtiments. Cela fait qu'il arrive que les projets calent si les promoteurs qui ont acquis les parcelles n'arrivent pas à ramasser l'argent suffisant pour construire et pour commercialiser. Il se crée alors une zone vide, sur un temps qui peut être long. Dans l'urbanisme américain, différent du nôtre parce que c'est un urbanisme essentiellement privé, les « crocodiles » sont ces terrains viabilisés par des entreprises ayant des projets de lotissements à l'extérieur des villes, qu'ils n'ont pu construire faute de pouvoir porter financièrement le projet jusqu'à son aboutissement. Ces zones sont intéressantes parce qu'elles ont une trame mais c'est tout, et qu'elles deviendront un ensemble bâti, ou non. Et probablement que cela n'est pas valable que pour notre époque.

LT Ce sont effectivement des considérations que nous commençons à mieux prendre en compte, ce qui fait changer notre vision de l'évolution des morphologies urbaines antiques et médiévales. En Gaule, au tout début de l'organisation de ces territoires par les autorités romaines, de nouvelles villes ont été créées, souvent, comme pour Évry, sans englober une agglomération antérieure. On se rend compte que ces villes ont presque toujours été surdimensionnées et que de nombreux espaces restent vides. Après, lorsque la ville s'étend, ce n'est pas forcément là où c'était « prévu » dans le schéma initial. Nous avons du mal à comprendre quels étaient les usages de ces espaces à proximité du cour de la ville, s'ils en avaient.

ST Est-ce qu'il est possible, archéologiquement parlant, d'identifier des usages de lieux qui les rapprochent des communs ou communaux? Des espaces dits vides à cause du peu de traces qu'on y décèle pourraient-ils être ces zones dédiées au glanage, au nourrissage des cochons etc. ? Le concept de communs est une question très présente aujourd'hui dans les études en sciences politiques et en économie, souvent en lien avec les études sur des environnements particuliers (alpage, forêt, rivière...). Mais pouvezvous les identifier autour des agglomérations? LT Les sources écrites mentionnent ces espaces prévus pour la communauté et cela nous mène à regarder différemment les franges urbaines antiques, qui ne sont pas encore la ville et 


\section{Pour donner}

une existence aux « espaces vides », il faut savoir parler de leurs usages, donc savoir en repérer les traces.

Laurence Tranoy

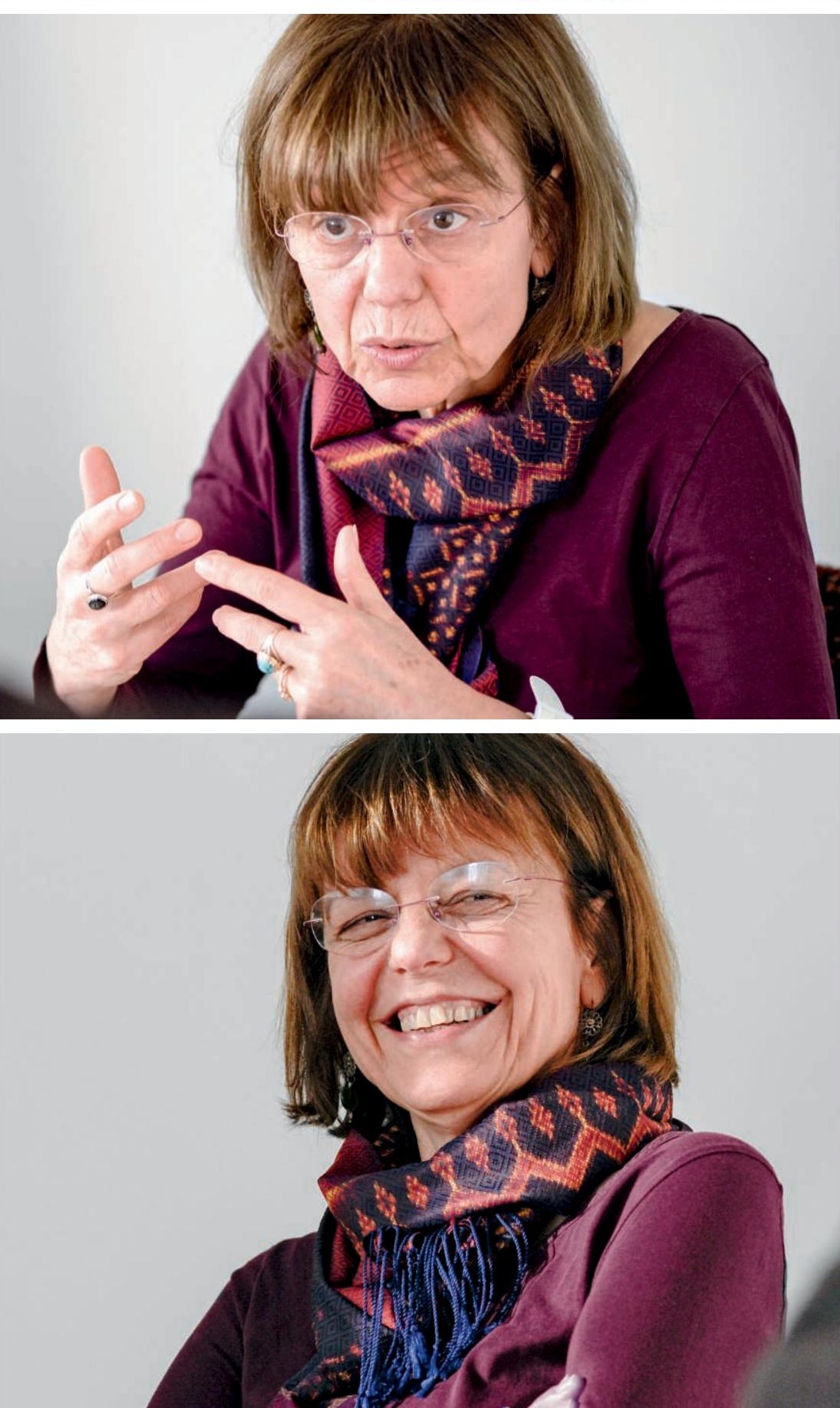


échappent à son schéma régulateur. Ce sont des espaces très intéressants parce qu'on sait qu'il s'y passe plein de choses : activités funéraires, cultuelles, artisanales, maraîchères, commerciales... On les a identifiées surtout grâce à l'épigraphie, à des inscriptions précisant l'organisation cadastrale, comme pour le cadastre d'Orange $^{\mathbf{6}}$. On commence à réfléchir plus systématiquement aux caractéristiques physiques de ces lieux, car ce sont aussi parfois des zones « à problème » : insalubres, marécageuses, inondables...

ST Les terrains sur lesquels je me suis penché sont, en effet, très influencés par la topographie, notamment par les voies d'eau et par les reliefs. C'est souvent à la rencontre de ces éléments importants du paysage que se trouvent des vides. Peut-être parce qu'il y a une certaine " perversité » de cette géométrie, ce sont des rencontres entre des métriques différentes, ou des questions environnementales à trancher, ce qui peut prendre un certain temps. Dans l'urbanisme d'aujourd'hui, par exemple, il y a cette idée qu'il faut dégager les alentours de fleuves, en faire des parcs urbains qui serviront de bassins de rétention en cas d'inondation. C'est cela qui est intéressant, cette double fonction pensée dès l'origine d'un espace public. Ce parti-pris arrive après toute une période pendant laquelle on a tenté de contrôler les fleuves, en faisant des rives en dur de plus en plus hautes.

LT Lors des fouilles à Lyon, menées conjointement avec des spécialistes de la morphodynamique fluviale ${ }^{7}$, on a pu suivre l'histoire des inondations des deux cours d'eau. On s'est rendu compte que les zones inondables étaient utilisées pour diverses fonctions (entrepôt, funéraire, artisanat, agriculture), fonctions, pour les périodes antiques, localisées en périphérie. ST À force de suivre l'évolution des terrains interstitiels sur lesquels je travaille, j'ai constaté que certains ont été réinvestis, rénovés, repris. Le plus intéressant est de voir qu'on y retrouve, ou prolonge, des traces des usages du temps où ce terrain était abandonné, une continuité entre la période dite « vide» et la période dite « pleine». Il y aurait donc quand même une prégnance des usages qui arriverait à passer au-delà de certaines étapes de construction dont on aurait pu penser qu'elles effacent tout ce qu'il y avait avant. C'est pour cela que je parle de la carrière d'un lieu, comme l'on parle de la carrière d'une personne, qu'elle soit professionnelle ou médicale.

LT C'est très archéologique comme démarche! Nous cherchons toujours à comprendre l'évolution d'un site mais lorsqu'elle n'apparaît pas évidente, on a peut-être trop tendance à parler de rupture au lieu de continuité. À la fin de l'Antiquité, certaines zones construites semblent abandonnées, des enceintes sont élevées... On a longtemps pensé que les villes antiques se rétractaient, et qu'au Moyen Âge, elles avaient une nouvelle vie. Or il y avait certainement des usages qui faisaient la continuité entre la ville antique et la ville médiévale mais qui n'ont pas été forcément perceptibles. Cette réflexion n'émerge que depuis que l'archéologie, un peu avant l'histoire ${ }^{\mathbf{8}}$, commence à s'affranchir des classifications périodiques. L'une des réponses pourrait se trouver dans le phénomène des « terres noires ». Elles ont d'abord été interprétées comme des terres végétales et on s'est reposé sur les environnementalistes pour en comprendre la signification car l'implicite était que le bâti est essentiellement minéral. Aujourd'hui, il semble que ces terres noires seraient des indicateurs de constructions en bois à toiture végétale, et/ou d'une gestion spécifique des déchets. Ces terrains que l'on pensait abandonnés à la fin de l'Antiquité pourraient donc avoir été investis, de façon informelle ou non. Il est significatif de constater que les rues continuent de jouer leur rôle de liaison et restent fréquentées au milieu de quartiers où les constructions semblent détruites ou abandonnées, ce qui ne veut pas dire non habitées. Le phénomène du squat est repérable archéologiquement: on sait repérer les traces de structures légères, de matériaux de récupération, de «bricolage » constructif à partir d'éléments bâtis encore en élévation, à usage d'habitation ou de travail. Et puis on y décharge des déchets, de matériaux de construction, par exemple. C'est cette fonction de zone de rebut, de marginalité, que l'on détecte le mieux.

ST De nos jours, la puissance publique décide souvent, en effet, que l'on va utiliser ces «bouts » de terrains pour s'occuper de choses qu'on ne voudrait pas avoir ailleurs. À Charenton, la ville s'est servi des terrains entre la Seine et l'autoroute pour collecter et pré-trier ses déchets. Mais ce sont aussi souvent des endroits où la société repousse une partie de la population qu'elle n'est pas capable de gérer dans ses espaces principaux. La zone non aedificandi des remparts de Paris, dès sa construction quasiment ${ }^{\mathbf{9}}$, est devenue une zone d'occupation illégale, dont les habitants ont été appelés les zoniers. C'était très habité ; les gens vivaient dans des constructions légères, telles que vous venez de les décrire pour l'Antiquité.

Le fort retour actuel des inégalités sociales génère de nouveau une occupation assez dense des bascôtés. Toutes ces utilisations-là sont parfaitement connues par les propriétaires des sites, qui les tolèrent car ce serait plus coûteux, ou plus compliqué, ou plus dommageable, en termes d'image, par exemple, de s'en occuper que de laisser faire. La gestion de ce type d'espace est de s'assurer soit que les terrains restent vides - si ces terrains sont fréquentés, et de fait, ils le sont, alors ils deviennent problématiques car ils se remplissent avec des constructions et avec des usages informels moins contrôlables - soit que les occupants restent dans les limites de la visibilité, c'est-à-dire de l'invisibilité. Les propriétaires fonciers, quels qu'ils soient, font 
tout pour qu'il n'y ait pas d'occupation en dur, car lorsque que les gens commencent à construire en dur, ils finissent par avoir des revendications. Il faut garder ces terrains dans une espèce d'entre deux pour qu'ils soient récupérables si et quand on en aura besoin ${ }^{\mathbf{1 0}}$. Dans les périodes de précarité, les terrains vides sont souvent exploités en jardins. Je pense que la question du jardinage est importante aujourd'hui dans tous ces espaces dont beaucoup ont été récupérés pour faire des jardins partagés, ce qui est une forme de retrouvaille avec le sol. C'est une autre façon d'habiter en ville. François Béguin ${ }^{\mathbf{1 1}}$, le paysagiste, dit que les premiers logements ouvriers construits dans les villes au passage du $\mathrm{XIX}^{\mathrm{e}}$ au $\mathrm{XX}^{\mathrm{e}}$ siècle l'étaient dans une ville pas encore complètement remplie, où restaient beaucoup de lieux pour de petites cultures. Même chose, plus tard, autour des premières barres. Il y avait donc, dans certaines phases de l'urbanisation, un possible contact avec la terre qui permettait au paysan qui migrait dans la ville de garder un peu de ses anciens modes de subsistance. En plus du boulot à l'usine, ils avaient aussi de quoi se nourrir par eux-mêmes, voire d'avoir un peu de revenu en sus. Mais l'avènement du salariat généralisé a coupé cette relation. Aujourd'hui on est peut-être en train de chercher à retrouver certaine forme de subsistance un peu moins monétarisée, un peu plus liée au sol. Ce lien au sol plus agraire par de nouveaux occupants de la ville ne doit pas être valable que pour nos époques. Est-ce que le jardinage laisse des traces visibles par l'archéologue?

LT Oui, ce type d'étude s'est développé à partir des années 1990, avec des résultats plus que probants $^{\mathbf{1 2}}$. Les spécialistes savent repérer tant les étapes et les procédés de construction des grands jardins que les plantations etc. Mais les études restent rares. On pourrait identifier l'usage comme jardin d'un terrain vide en milieu urbain, au moins en stratigraphie. Les villes pérennes sont faites de ces espaces qui ont eu plusieurs phases architecturées, entrecoupées de périodes de destruction due à des causes diverses, d'abandon, de réinvestissement... qui parfois modifient les relations entre les lieux et leurs usages. Quant aux villes antiques qui ont cessé d'exister, on a l'impression qu'elles ont été abandonnées très rapidement au $\mathrm{III}^{\mathrm{e}}$ siècle. À un moment donné, elles ont disparu de la surface, le lieu devient des champs. C'est l'échelle de temps que l'on ne saisit pas vraiment. Ces villes ont dû péricliter petit à petit, elles se sont effilochées... mais sur combien de temps?

ST C'est un déclin peut-être moins rapide qu'on ne le pense. Et l'arrivée d'un autre type de population, d'un autre type d'habitat. L'évolution d'un terrain est faite de ruptures et de continuités, mais effectivement les continuités sont peut-être plus importantes qu'on le pense. Et si on arrive à les identifier, elles permettent de tracer des carrières, des généalogies d'usages. Et ces usages durent plus longtemps que on ne l'aurait suspecté. Par exemple, sur une jetée que j'ai étudiée aux États-Unis, un jardin potager pirate, entretenu pendant quelques années, a été incorporé dans un jardin dit communautaire une fois que l'espace de la jetée a été refait. Au début, il était porté par les mêmes gens que le jardin pirate, puis ils se sont fait éjecter, le contrôle du terrain est passé à d'autres. Mais s'il y a un jardin dans ce lieu aujourd'hui, c'est grâce à ceux qui en avaient un dans le terrain abandonné d'avant, même s'il n'est pas exactement au même endroit. C'est bien une trace mais elle n'est pas la trace matérielle de l'existant d'avant, c'est la trace de l'usage d'avant qui a été préservée, mais un peu déplacée. À l'échelle archéologique, c'est sans doute plus difficile qu'à celle de mes recherches (sur vingt ans) de voir comment ce type de traces restent. Mais peut-être y a-t-il d'autres façons de voir? à Charenton, les

exploitants du pipeline,

craignant que les

passaient réguliè

et la police, tous les

jours. Un recensemen

de ces habitants a eu lieu,

mais il n'a pas figuré dan

les chiffres municipaux.

11. F. Béguin,

"Vagues, vides, verts",

Le Visiteur, $\mathrm{n}^{\circ} 3$.

12. Archéopages, 37,

« Jardins », 2013. 